\title{
Postoperative obstructing laryngeal edema in patients with diffuse idiopathic skeletal hyperostosis of cervical spine -A report of two cases-
}

\author{
Young-Soon $\mathrm{Kim}^{1}$, Jeong Jin Lee ${ }^{1}$, Yang Hoon Chung ${ }^{1}$, Eun Sang Kim², and Ik-Soo Chung ${ }^{1}$ \\ Departments of ${ }^{1}$ Anesthesiology and Pain Medicine, ${ }^{2}$ Neurosurgery, Samsung Medical Center, Sungkyunkwan University School of \\ Medicine, Seoul, Korea
}

Two cases were reported in which severe postoperative laryngeal edema were developed after the operation of diffuse idiopathic skeletal hyperostosis (DISH) of cervical spine. In the first case, sudden airway obstruction was developed in the general ward 6 hour after uneventful decompression surgery for osteophyte. In the second patient, an elective preoperative tracheostomy was performed before surgery but the tube could not be removed for 2 months because of laryngeal edema and decreased vocal cord mobility. It should be emphasized that this airway problem can develop during the postoperative as well as the preoperative period, especially in the case of anterior cervical spine surgery. (Korean J Anesthesiol 2011; 60: 377-380)

Key Words: Airway obstruction, Cervical spine, Diffuse idiopathic skeletal hyperostosis.

Degenerative conditions as well as diffuse idiopathic skeletal hyperostosis (DISH), so called Forestier's disease, commonly lead to anterior cervical osteophytes. While most patients are asymptomatic, a small percentage develop dysphagia and airway complaints $[1,2]$. The literatures emphasizes that anterior osteophytes on the cervical vertebra combined with subglottic stenosis, results in distortion of the airway and leads to unexpected difficulties during intubation [3]. This airway problem can develop during the postoperative or preoperative period. We experienced 2 cases in which severe laryngeal edema developed after uneventful decompression surgery for osteophyte.

\section{Case Reports}

\section{Case 1}

A 73-year-old man $(168 \mathrm{~cm}, 59 \mathrm{~kg})$ presented at our institution complaining of neck stiffness and dysphagia that had been slowly increasing over the last 2 years. He had been diagnosed with atrial fibrillation and stable angina, and undertook percutaneous coronary intervention (PCI) 6 months prior to the surgery. He had undergone anticoagulation therapy, which was changed to heparin 3 days before surgery. On physical examination, the patient was alert and afebrile; and his vital

Received: October 6, 2010. Revised: November 30, 2010. Accepted: December 6, 2010.

Corresponding author: Jeong Jin Lee, M.D., Ph.D., Department of Anesthesia and Pain Medicine, Samsung Medical Center, Sungkyunkwan University School of Medicine, 50, Ilwon-dong, Kangnam-gu, Seoul 135-710, Korea. Tel: 82-2-3410-2467, Fax: 82-2-3410-0361, E-mail: 1jj6625@ gmail.com

(c) This is an open-access article distributed under the terms of the Creative Commons Attribution Non-Commercial License (http:// creativecommons.org/licenses/by-nc/3.0/), which permits unrestricted non-commercial use, distribution, and reproduction in any medium, provided the original work is properly cited. 

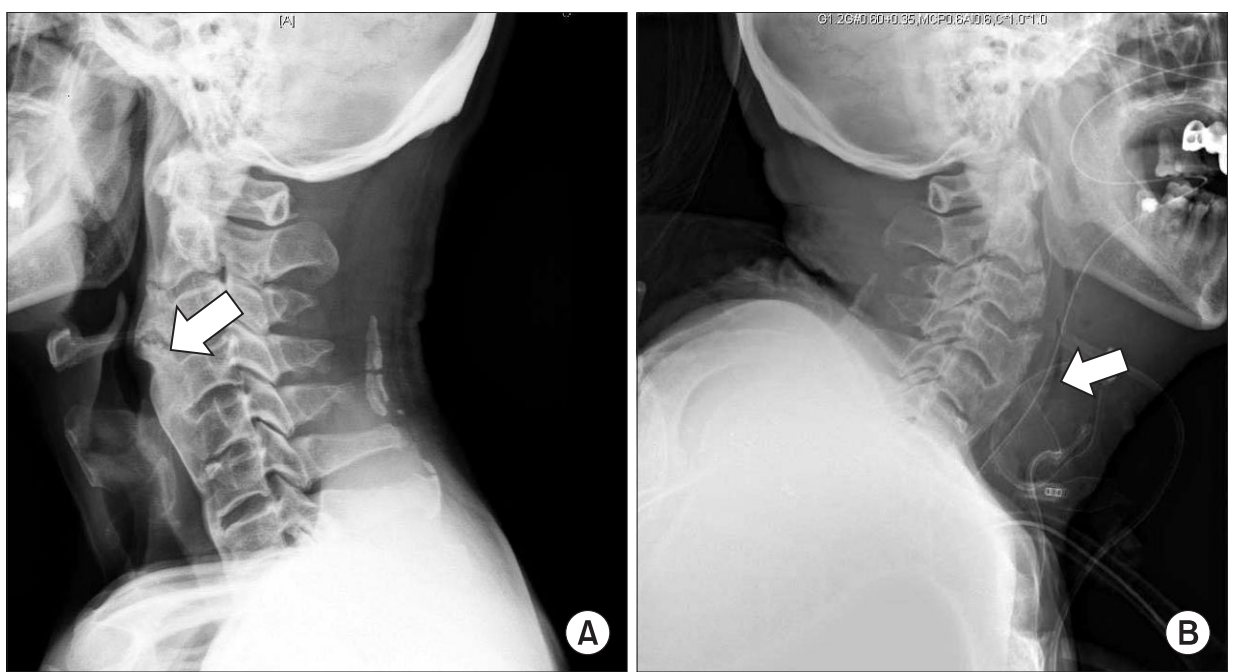

Fig. 1. Lateral view of cervical spine of patient 1 at (A) preoperative: diffuse osteophyte at C2-4 level (arrow). (B) postoperative lateral cervical wiew after 2nd surgery shows severe narrowing of air passage (arrow) above tracheostomy site.

signs were stable. Oral examination was unremarkable. Blood chemistry revealed no remarkable findings. Heparin was discontinued at preoperative midnight. Lateral radiography of the cervical spine demonstrated extensive osteophyte formation from C2 to C4 (Fig. 1A). A preoperative neck computed tomography (CT) revealed slight narrowing of the airway at the level of epiglottis, a finding compatible with Forestier's disease. General anesthesia was induced with thiopental sodium $250 \mathrm{mg}$ and rocuronium $50 \mathrm{mg}$ and endotracheal tube was inserted with lightwand without difficulty. The intraoperative findings using an anterolateral transverse approach included a hard bony mass at the level of C2-4 and compression of the esophagus and trachea. Decompression of the osteophyte was performed with a drill for 135 minutes. The patient was transferred to the postanesthetic care unit (PACU) following the operation. In the PACU the endotracheal tube was removed after full assessment of consciousness and muscle power. The patient was transferred to the general ward and had no complaints until 6 hour after the completion of surgery. At that time, patient complained of dyspnea and sudden respiratory compromise and cardiac arrest were developed. Emergent endotracheal tube insertion was attemped but the hypopharyngeal structure could not be identified because of severe laryngeal edema. Emergency tracheostomy was done with an ID 7.0 tube. With the impression of postoperative bleeding, emergent surgery was arranged. However, there was no bleeding at the operative site. Instead, the connective tissue and mucosa were severely edematous. Lateral radiography of the cervical spine demonstrated extensive soft tissue edema and airway narrowing (Fig. 1B). There was no evidence of remaining osteophyte. The patient's brain was damaged and discharged with serious neurologic sequelae.

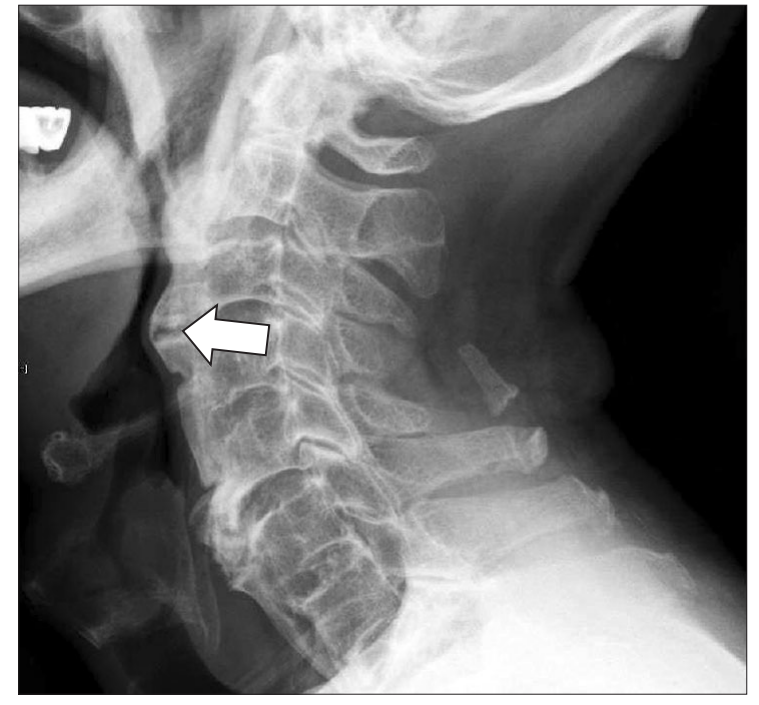

Fig. 2. Preoperative lateral view of cervical spine of patient 2 shows protruding mass of C2-3, 3-4 (arrow).

\section{Case 2}

A 74-year-old male patient ( $168 \mathrm{~cm}, 53 \mathrm{~kg}$ ) visited our institution complaining of difficulty in swallowing for the past 8 years and the frequent occurrence of aspiration pneumonia for the past 1year. Lateral radiography of the cervical spine demonstrated extensive osteophyte formation from C2 to C4 (Fig. 2) and decompression of the osteophyte was planned. His medical history was significant for diabetes. He had been diagnosed with stable angina but rejected PCI. Preoperative electrocardiogram and echocardiogram revealed tolerable status. Blood chemistry revealed hyperglycemia, but hepatic and renal function and coagulation were normal. On physical examination, the patient was alert and afebrile; and his vital 

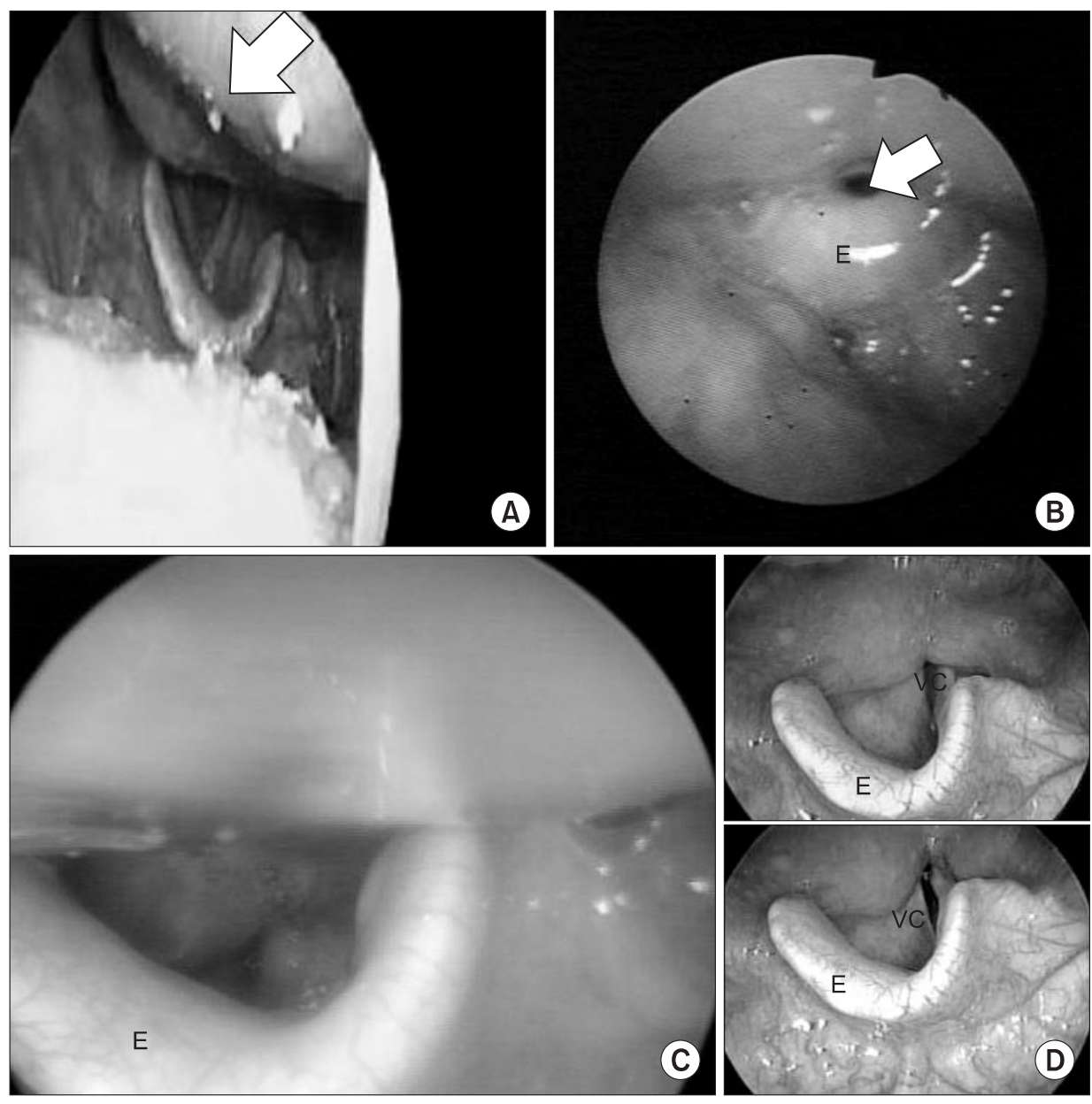

Fig. 3. Indirect laryngoscopic view of patient 2 at (A) preoperative: protruding mass (arrow) partially hide vocal cord. (B) POD\#2: airway (arrow) is narrowed due to generalized edema of epiglottis (E) and hypopharynx. (C) POD\#29; slightly decreased edema of epiglottis (E). But large amount of secretion disturbs clear view. (D) POD\#59 (E: epiglottis, VC: vocal cord). signs were stable. Oral examination was unremarkable and neck extension was normal. He had minimal dyspnea in the supine position. Indirect laryngoscopy revealed a large hard mass protruding from the posterior wall of the hypopharynx that concealed the arytenoids cartilage (Fig. 3A). A preoperative neck CT showed slight narrowing of the airway at the level of the epiglottis. Vocal fold mobility was preserved. No narrowing of the subglottic region was observed when flexible endoscopy was performed. Preoperative tracheostomy was done under local anesthesia and general anesthesia was induced with thiopental sodium $250 \mathrm{mg}$ and rocuronium $50 \mathrm{mg}$ and maintained with sevoflurane. Complete decompression of the osteophyte was performed for 210 minutes using an anterolateral transcervical approach, which proceeded without any complications. The postoperative course was uneventful. On the second postoperative day, indirect laryngoscopy was performed by an otolaryngologist to decide if removal of the tracheostomy cannulawas appropriate. However, this examination revealed significant laryngeal edema including the epiglottis and posterior laryngeal wall (Fig. 3B). On 29th postoperative day, flexible endoscopic examination was performed again and copious secretion, decreased vocal cord mobility and narrowing of the upper airway were observed (Fig. 3C) and the patient was discharged on the $33^{\text {rd }}$ postoperative day with a double cannula having a speech valve. On the 59th postoperative day, the tracheostomy cannula was removed (Fig. 3D) at the outpatient clinic without any complication.

\section{Discussion}

DISH is an ossifying diathesis leading to bone formation in spinal and extraspinal sites, paravertebral osteophyte formation and ligamentous calcification and ossification. Ossification of the anterior longitudinal ligament is common, might be discontinuous; and it is often more marked in the thoracolumbar spine than elsewhere. However, isolated and predominant cervical spinal involvement may occur [3]. In general, DISH requires treatment only when symptomatic. Conservative treatment with non-steroidal antirheumatic drugs and steroids may provide some relief in cases of mild dysphagia. In severe cases or cases with airway obstruction, surgical excision of osteophytic masses is the treatment of choice [4]. 
Difficult intubation resulting from anatomical abnormalities of the cervical spine is rare, but can result in the deterioration of the patient condition. Preoperative radiological evaluation may be useful in assessing the airway in patients deemed to be at risk for difficult intubation. Careful clinical evaluation of the airway prior to surgery and a predetermined approach for handling unexpected difficult intubation have been emphasized [3]. Fiberoptic-assisted awake intubation is the safest choice in DISH patients when difficult intubation is suspected [5]. In our first case, intubation was performed with a lightwand without difficulty, but the second patient required preoperative tracheostomy.

Most literature suggests that osteophytectomy can release dysphagia or airway obstruction [6]. Postoperative laryngeal edema has not yet been emphasized. Carlson et al. [7] performed a retrospective chart review on 9 patients who underwent osteophytectomy for dysphagia or airway complaints, including 6 patients with DISH, during the last decade (19982008). The postoperative course of these patients was not eventful in all 9 patients. The only postoperative airway event after cervical spine surgery in the literature was a postoperative hematoma case reported by Miake et al. [8]. In our first case, we also thought that asphyxia developed because of postoperative hematoma; however, no hematoma was found at the operative site. We found severe laryngeal edema with a pinhole sized airway space in the second case (Fig. 3B). Marks et al. reported 1 patient who required preoperative tracheostomy and could be decannulated 4 weeks after decompression surgery [4]. This shows that postoperative airway edema can occur and should be managed carefully especially in patients with preoperative laryngeal edema.

Preoperative laryngeal edema is not rare in DISH patients. It was suggested that the tissue reaction around the osteophyte spikes leads to fibrosis and adhesions, preventing the normal gliding movements of the esophagus and larynx during deglutition [4]. In our cases it appeared that chronic mechanical irritation of the retrocricoarytenoid area from rolling over prominent and spiky osteophytes during each swallow resulted in a chronic inflammatory edema that narrowed the hypopharynx and surgical irritation worsened the inflammation. Moreover the location of osteophyte was in the direct posterior part of the airway and the airway was easily compromised with a small hematoma or a small amount of edema [8].

In conclusion, postoperative airway obstruction can develop in patients who undergo decompression surgery for DISH. To prevent this complication, it is important to understand this condition. We should have alerted that such a complication may occur after anterior cervical spinal surgery. It is also important to be aware that intubation of such a case becomes difficult once the trachea has become compressed and curved because of a hematoma or edema.

\section{References}

1. Kmucha ST, Cravens RB Jr. DISH syndrome and its role in dysphagia. Otolaryngol Head Neck Surg 1994; 110: 431-6.

2. Giger R, Dulguerov P, Payer M. Anterior cervical osteophytes causing dysphagia and dyspnea: an uncommon entity revisited. Dysphagia 2006; 21: 259-63.

3. Crosby ET, Grahovac S. Diffuse idiopathic skeletal hyperostosis: an unusual cause of difficult intubation. Can J Anaesth 1993; 40: 54-8.

4. Marks B, Schober E, Swoboda H. Diffuse idiopathic skeletal hyperostosis causing obstructing laryngeal edema. Eur Arch Otorhinolaryngol 1998; 255: 256-8.

5. Ozkalkanli MY, Katircioglu K, Ozkalkanli DT, Savaci S. Airway management of a patient with Forestier's disease. J Anesth 2006; 20 : 304-6.

6. Nelson RS, Urquhart AC, Faciszewski T. Diffuse idiopathic skeletal hyperostosis: a rare cause of dysphagia, airway obstruction, and dysphonia. J Am Coll Surg 2006; 202: 938-42.

7. Carlson ML, Archibald DJ, Graner DE, Kasperbauer JL. Surgical management of dysphagia and airway obstruction in patients with prominent ventral cervical osteophytes. Dysphagia 2011; 26: 34-40.

8. Miake G, Arizono T, Yamaguchi T, Akune H. Case of critical complication after surgery for ankylosing spinal hyperostosis. Fukuoka Igaku Zasshi 2009; 100: 104-7. 